\title{
Reputation-based Policies that Provide the Right Incentives in Peer-to-Peer Environments*
}

\author{
Thanasis G. Papaioannou ${ }^{\mathrm{a}}$, George D. Stamoulis ${ }^{\mathrm{a}}$ \\ a Department of Informatics, Athens University of Economics and Business (AUEB), 76 Patision Str., \\ 10434 Athens, Greece. \{pathan, gstamoul\}@aueb.gr
}

Peer-to-peer are popular environments for exchanging services. A reputation mechanism is a proper means of discovering low-performing peers that fail to provide their services. In this paper, we present an in-depth and innovative study of how reputation can be exploited so that the right incentives for high performance are provided to peers. Such incentives do not arise if peers exploit reputation only when selecting the best providing peer; this approach may lead high-performing peers to receive unfairly low value from the system. We argue and justify experimentally that the calculation of reputation values has to be complemented by proper reputation-based policies that determine the pairs of peers eligible to interact with each other. We introduce two different dimensions of reputation-based policies, namely "provider selection" and "contention resolution", as well as specific policies for each dimension. We perform extensive comparative assessment of a wide variety of policy pairs and identify the most effective ones by means of simulations of dynamically varying peer-to-peer environments. We show that both dimensions have considerable impact on both the incentives for peers and the efficiency attained. In particular, when peers follow fixed strategies, certain policy pairs differentiate the value received by different types of peers in accordance to the value offered to the system per peer of each type. Moreover, when peers follow dynamic strategies, incentive compatibility applies under certain pairs of reputation-based policies: each peer is provided with the incentive to improve her performance in order to receive a higher value. Finally, we show experimentally that reputation values can be computed quickly and accurately by aggregating only a small randomly selected subset of the ratings' feedback provided by the peers, thus reducing the associated communications' overhead.

Keywords: reputation, policies, incentives, performance, randomized aggregation

\section{Introduction}

The peer-to-peer paradigm gains increasing acceptance in the information society as an overlay environment for exchanging services, such as digitized content. The value of such a service for the client peer depends on the performance level of the peer providing it, which may be unacceptably low. The reason for this can be either the peer's hidden type (i.e., her strategy on service provision) or her hidden quality (i.e., her actual ability to provide the service). Reputation can be a proper means of revealing low-performing peers in peer-to-peer electronic environments (as well as in markets), if it is defined appropriately and

*The present work was partly funded by the IST project MMAPPS (IST-2001-34201). A preliminary version was presented in the IEEE CCGRID International Global and P2P Computing Workshop, April 2004, Chicago, USA. calculated accurately [1], [2]. However, the accurate calculation of the reputation value by itself may not be adequate a mechanism to improve the achievable efficiency of high-performing peers (i.e. their satisfaction from the services provided to them) and to offer the right incentives for peers to provide services of high quality to others.

This paper provides an in-depth and innovative analysis of how reputation can be exploited in a way that it provides the right incentives to peers regarding their strategy for providing services. Such incentives do not arise under the straightforward approach for peers to exploit the reputation metrics, i.e. employ them only to select the highest-reputed providing peer. Specifically, this straightforward approach leads to unexpectedly low efficiency for high-performing peers when no other reputation-based incentive mechanism is 
employed in the peer-to-peer system. However, the quality of the services received by the various peers should be differentiated according to their contribution to the overall set of services of acceptable quality. This is required in order for the right incentives regarding performance for service provision to be provided to peers and for fairness reasons. Such incentives should also be provided to peers that follow strategies that are individually rational. That is, it should be beneficial for such peers to improve their performance regarding service provision. In this paper, we argue and justify experimentally that the calculation of the reputation values has to be complemented by reputation-based policies that determine the pairs of peers eligible to interact with each other. We introduce two different dimensions of reputationbased policies, namely "provider selection" and "contention resolution", as well as specific policies for each dimension. Reputation-based policies result in a cycle including calculation and exploitation of reputation. We prove that this cycle greatly affects the speed and the accuracy of revelation of hidden information.

We perform an extensive series of experiments in a simulation environment under the most stringent realistic assumptions. Experimental results show that both policy dimensions have a significant impact to both the incentives provided regarding performance in service provision and to the efficiency enjoyed by high-performing peers. Also, we identify the most effective policy pairs (i.e. combinations of a provider selection policy with a contention resolution policy) in terms of the efficiency enjoyed by high performing peers and the incentives provided for successful service provision in a variety of cases: a) for a dynamic environment with a continuously renewed population of peers that follow fixed strategies, b) when undesired exploitation (i.e., "milking") of reputation is employed, and c) when individually rational strategies are employed by peers. In particular, when peers follow fixed strategies, certain policy pairs differentiate the value received by different types of peers in accordance to the value offered to the system per peer of each type. Also, the same policy pairs limit significantly the potential for undesired exploitation of reputation.
Moreover, when peers follow dynamic individually rational strategies, incentive compatibility applies under certain pairs of reputation-based policies: each peer is provided with the incentive to improve her performance for providing services successfully to others in order to receive a higher value from the system. Then, we discuss the implementability of the various reputationbased policies in practice.

Last, we deal with the reduction of the communication (and processing) overhead for aggregating the ratings' feedback in order to accurately calculate reputation values. We propose that just a small randomly selected subset of the complete ratings' information has to be aggregated. We show experimentally that this aggregation approach does not essentially lead to any degradation of the speed or the accuracy of the calculation of the reputation values, even if the population of peers is renewed with a high rate.

The remainder of this paper is organized as follows: In Section 2, we briefly discuss how reputation can be calculated by aggregating peers' ratings. In Section 3, we comment on the straightforward approach of using reputation in a peerto-peer system. In Section 4, we introduce two different policy dimensions and propose specific reputation-based policies for each dimension. In Section 5, we present experimental results on the effectiveness of our reputation-based policies in the efficiency of high performing peers and in the incentives provided when a variety of strategies is followed by peers. In Section 6, we argue for the employability of the proposed reputation-based policies in real peer-to-peer systems. In Section 7, we introduce our approach for randomized aggregation of the ratings' feedback, we evaluate it experimentally and discuss its employability. In Section 8, we present a detailed comparison of our work with other related works, and, finally, in Section 9 , we provide some concluding remarks.

\section{Calculation of Reputation}

Each peer may either succeed or fail in offering a service to another peer; the likelihood of each outcome depends on several factors (see Subsection 5.2). Actually, only the outcome of each 
transaction is of interest to a peer to whom a service was provided, rather than the hidden reason for this outcome. After observing the outcome of her transaction, a client peer rates the providing one for her performance. Throughout the paper, we assume that peers truthfully report their evaluations for the performance of others. Mechanisms that ensure truthful reporting are proposed in [3] and [4]. Binary rating (i.e., success vs. failure) is known to be adequate for calculating a reputation value that is representative of the expected outcome of a transaction with a specific peer [1], [2]. The aggregation of all ratings that a specific peer got for the services she provided in a single reputation value is also important for practical reasons (e.g., the storage overhead is reduced). According to [1], Bayes' rule is an efficient aggregation function, which can be applied if: a) peers are classified in types, each having a fixed probability for successfully providing services, and b) there are defined initial beliefs on these probabilities and on the proportions of the types in the peer-to-peer system. In particular, reputation of each peer then expresses the a posteriori belief that she belongs to a certain type given the history of her service provisions. If the distribution of peer types in the peer-to-peer system is renewed dynamically and/or peers follow dynamic strategies over time (thus altering their probability of success) rather than fixed ones, then Beta aggregation [2] is more appropriate. Indeed, Beta aggregation does not depend on any prior beliefs for the distribution of peer types in the system. According to this approach, each peer's reputation equals the fraction of the "weighted number" of her successful service provisions over the "weighted total number" of her service provisions, with the weight of each service provision being a negative exponential function of the elapsed time. In the simulation experiments of Section 5, we use both Bayesian and Beta aggregation functions.

\section{The Straightforward Approach of Ex- ploiting Reputation}

Assume that a reputation mechanism is employed in a peer-to-peer environment that leads to the accurate calculation of a reputation value for each peer. This value reflects her probability to successfully provide her service the next time requested. Throughout the paper, we assume that reputation values are safely stored by the reputation system. Reputation limits the risk for a peer to fail in a service transaction. But, how would peers use these reputation values? Consider a peer requesting a service. We assume that each client peer aims to maximize the utility she obtains when being served. If all successfully provisioned services have the same value for the client peer, then she would select among other peers that offer the requested service the one that has the maximum reputation value. On the other hand, this same peer is indifferent in selecting among requesting peers whom to serve with her limited resources, if no other mechanism than announcement of reputation values is in place.

The above approach towards selecting transacting peers can be considered as the straightforward approach for employing reputation. However, it appears that a high-performing peer is thus unfairly "punished": she receives equal benefit from the peer-to-peer system as other peers that have a lower performance level than him, regardless of her high contribution to the total value of the peer-to-peer system. Clearly, this approach of peers to exploit the reputation metrics provides wrong incentives both to high- and to low-performing peers. A high-performing peer is motivated to lower her performance, while a lowperforming one is motivated to keep her performance at the same level and continue to free-ride. These incentives lead to a market of "lemons", and possibly to the gradual collapse of the peerto-peer system. Thus, an appropriate reputationbased policy that provides the right incentives to peers, altering this default response, has to be introduced in the peer-to-peer services environment. Such a reputation-based policy should result to higher benefit enjoyed by peers with higher performance, thus providing a clear incentive for peers to improve their performance.

\section{Reputation-Based Policies}

We classify the reputation-based policies into two dimensions: "provider selection" and "con- 
tention resolution". The former regulate the selection of the providing peer among those offering the same service; the latter regulate the selection, among all peers requesting a service from the same providing peer with limited resources, of the peer to be actually served. The assumption of a peer's limited resources is a realistic one and can be related with each peer's upstream capacity, her availability of CPU cycles, etc. Next, we present certain policies for each dimension. Note that we introduced "contention resolution" dimension and its specific reputation-based policies, published in [5]. Such policies also appeared in two subsequent published papers [8], [11] (see Section 8).

\subsection{Provider Selection Policies}

Highest Reputation: If a reputation metric for performance is available in the peer-to-peer system, then the most reasonable policy for each peer is to select, among peers that provide the requested service, the one with the highest reputation value. This policy was used for experiments with reputation in [6].

Comparable Reputation: A policy named "Peer-Approved" was studied in [7]. According to that policy, peers can receive services only from other ones with lower or equal rating. For a peer that improves her performance, and thus her reputation value, this policy increases the probability to find the services requested. However, the received quality of such a peer is questionable, as she may select services from peers with lower reputation values, and hence, lower performance. We propose a different policy, referred to as "Comparable Reputation", whereby peers are able to request services only from peers that have reputation values comparable to theirs, i.e. with a pre-specified maximum difference. The underlying idea of this policy is the matching of the performance level offered by a peer with the performance level provided to him. Thus, this policy results in layered communities. Peers of the same layer exchange services of similar quality. If there are high-performing peers in the peer-to-peer system, then the quality of offered services is high in the top layer. On the other hand, the services offered in the lowest layer can be useless or even harmful for other peers.

Black List: This policy expels from the system peers that have reputation values below a certain threshold. Specifically, peers offering services of low quality consistently for a certain period are excluded from the set of eligible providing peers. Therefore, this policy improves the quality offered to the remaining peers.

\subsection{Contention Resolution Policies}

In a real peer-to-peer system, a new service request may arrive at a peer that already serves some previous requests. Then, due to her limited resources, the peer has to decide whether to serve or block or queue the new request at the expense of quality of those already being served. This decision is made according to a contentionresolution policy, which has thus to be employed. In fact, in systems where high performing peers are considerably fewer than low performing ones and provider selection policies are employed, contention for the resources of the former arises very frequently. The relevant part of our model is somewhat simplified. Time is assumed to be slotted. Multiple requests may arise at a peer at the beginning of each time slot. Only one of these requests is served and service duration lasts for one time slot. This model does capture the issue of limited resources at a peer that force her to successfully serve only limited number of requests at a time slot.

Highest Reputation: According to this policy (see also [8]), the peer with the highest reputation value is the one selected to be served by a peer among those simultaneously requesting a service from the latter and thus contending for her resources. This policy assigns absolute priority to peers with the highest reputation values. (Ties are resolved by means of a symmetric randomized rule.) Thus, a peer with a high reputation value is very likely to be provided the service even when she is contending with others. However, the outcome of service provision depends on the provider selection policy that is employed in the peer-to-peer system. If this policy is in use and there is serious contention for resources, then peers with low reputation values will essentially be offered no services. 
Probabilistically Fair with respect to Reputation: According to this policy (to be referred to as "Probabilistically Fair"), the peer to be served is selected according to the following rule: among the peers $j$ that request the same service from a particular peer, the probability for a specific peer $i$ to be selected equals $R_{i} / \sum_{j} R_{j}$, where $R_{j}$ is the reputation value of peer $j$. Note that peers with high reputation again have an advantage over the others. However, under the present policy, peers with low reputation values always have a positive (possibly small) probability of receiving some services regardless of whom they contend with. Finally, in case that all peers contending for a certain resource have equal reputation values, the two contention resolution policies coincide.

\subsection{Discussion}

A contention resolution policy determines the probability that a peer will be provided a service, in the presence of contention. The probability that a service provision is indeed successful depends on the provider selection policy, which however influences also the set of peers contending for the same service. Thus, depending on the specific kind of services that are offered in the peerto-peer environment and the relative importance between the quality and the quantity of services exchanged, the proper pair of provider selection and contention resolution policies should be employed in the system (see Subsection 5.2). Therefore reputation-based policies determine the pairs of peers that are eligible to interact. The ratings' feedback on the performance of providing peers is sent by the client ones to the reputation system either immediately or after a number of completed transactions. The reputation values of the respective peers are updated on the basis of this feedback. However, these updated values determine the new pairs of peers that are eligible to interact. These interactions will result in additional ratings' feedback, etc. Thus, when reputation-based policies are employed in the peer-to-peer system, a cycle of reputation information is formed. The selection of the pair of reputation-based policies employed greatly influences how the reputation mechanism will lead to the revelation of the hidden true information regarding each peer's type as well as the incentives provided to peers regarding their performance on offering their services successfully, as shown in the next section.

The initial reputation value $h_{0}$ of each new entrant peer has to be small, in order to limit the incentive for name changes [10]. That is, if $h_{0}$ were high, then each peer would have the incentive to drop her pseudonym and obtain a new one, thus clearing her low-performance records. New entrant peers or low-performing ones are still able to attract transactions (i.e. and potentially to improve their reputation), despite the employment of provider selection reputation-based policies, as a certain requested service may be found only to such peers. For simplicity, we have not considered an extra mechanism forcing a peer to keep on contributing to the system, particularly after having established a high reputation. In certain peer-to-peer systems, this is mandatory. If not, the reputation could be decreased with time in order the right incentives for high contribution to be maintained.

\section{Simulation Experiments and Analysis of Results}

\subsection{The Simulation Model}

We have specified a simulation model of a peerto-peer system where services of a certain kind are exchanged among peers. The corresponding simulation environment was implemented in Java. Similarly, as in [1], there are two considerably different types of peers in this system: altruistic and egotistic. The type of each peer is private information, i.e. it is known only to the peer himself. Each peer exhibits (either intentionally or inherently due to limitation of her resources) a strategy regarding her performance in her service provisions; this strategy depends on the peer's type and it does not vary during the lifetime of a peer. We assume that the strategy of a peer of a certain type along with other unpredictable factors, e.g. network congestion conditions or bandwidth constraints due to the capacity of the receiver, result in a certain probability of successful service provision for each type. In particular, each altruistic (resp. egotistic) peer provides a service successfully with a high probability $\alpha=0.9$ (resp. 
with a low probability $\beta=0.1$ ). Time is assumed to be slotted. A time slot is taken to be the minimum time period between two successive service requests by the same peer. Each service provision lasts for one time slot. Since rejected requests are not queued (see Subsection 4.3), successive service provisions by the same peer are taken as independent.

According to [9], in real situations, the fraction of free-riders (or egotistic) is nearly $70 \%$ of the population in a peer-to-peer community. In order to derive reliable conclusions, we have studied the effectiveness of the various reputation-based policies under more stringent conditions. In particular, we have assumed that the vast majority of peers $(90 \%)$ are egotistic. Furthermore, the population of peers is assumed to be renewed according to a Poisson process with mean rate $\lambda=10$ peers/time slot, while the total size $N$ of the population is kept constant with $N=1000$. That is, each peer is assumed to live in the peer-to-peer system for a period determined according to the exponential distribution with mean $N / \lambda$. When a peer leaves the system a new entrant of the same type takes her place, using a new pseudonym. A reputation system is employed with the reputation value for a peer being associated to her pseudonym. Each peer sends feedback to the reputation system on the performance of other peers based on the outcome of the services provided by the latter to the former. Feedback is considered truthful and depends only on the outcome of a service provision, which is assumed to be observed in an objective way. The initial reputation value $h_{0}$ is small: $h_{0}=0.1$. The ratings are converted into reputation values using Bayes' rule, in Subsections 5.2 and 5.3, as in these cases the strategy followed by peers is static. Hence, reputation expresses the probability that a peer is of the altruistic type given her records of service provisions. Note that the experiments of Subsections 5.2 and 5.3 were also run using the Beta function for the calculation of reputation values and the results were similar. This fact indicates that the conclusions regarding the necessity and the effectiveness of reputation-based policies apply provided that the aggregation function accurate reveals the hidden type of peers. However, the Beta aggregation function is used for the calculation of reputation values in the experiments of Subsections 5.4 and 5.5, as peers are there assumed to employ dynamic strategies.

Every peer requests a service with a certain probability $r=0.5$. This value of $r$ was selected in order for the reputation values to converge to equilibrium in a reasonable number of time slots. $r$ has no impact on the relative effectiveness of reputation-based policies. In the experiments presented in this section, service availability is Zipf-distributed. That is, assuming that services are ranked with respect to their popularity, a service with rank $x$ is found at a certain peer with probability $x^{-1}$. The same experiments were also conducted having services uniformly distributed over the population of peers, and the results were similar. The underlying service discovery model is fully distributed, while a unique globally retrievable reputation value is available for each of the peers. Different reputation and service discovery models could also be used. These would have no impact on the obtained results, as long as reputation can be stored and retrieved by all peers. A peer can serve only one other peer at each slot due to her limited resources. Denial of service in our model due to limited resources, corresponds to realistic situations of temporary unavailability of the service, e.g. due to congestion in the network or in a server. Reputation-based policies are employed in most of the experiments. When this is not the case, peers are taken to select their transaction party according to the straightforward approach described in Section 3.

One of the main objectives of the experiments is to assess the efficiency attained in this peer-topeer system under the various policies. Efficiency can be measured by: the ratio of successful transactions per peer over either i) the average number of initiated services (i.e., services found and started after contention resolution) or ii) the average number of service requests. The corresponding efficiency measures will be referred to as a) success ratio among started services, which equals the total number of services offered successfully to peers of a specific type divided by the total number of services that started to be provided to them and b) success ratio among requested ser- 
vices, which equals the total number of services offered successfully to peers of a specific type divided by the total number of services that were requested by these peers. We are mostly interested in the efficiency enjoyed by altruistic peers. To motivate these efficiency measures first note that only successful transactions provide value to client peers. In order for a peer to be provided the right incentives for performance, she should receive value according to her offered performance. Thus, altruistic peers should be offered high value by the peer-to-peer system, in order to stay and keep on offering value to the system. On the other hand, egotistic peers inherently offer low value to the peer-to-peer system, and thus the value provided to them by the system is not an important measure for efficiency. Nevertheless, on a per peer basis, this value should be lower than that provided to altruistic peers; this is also a fairness requirement. Efficiency is affected by the fast and accurate revelation of the hidden type of peers, which depends on the reputation-based policies employed that affect the reputation cycle discussed in Subsection 4.3.

\subsection{Effectiveness of Reputation-Based Policies}

If no reputation system were employed in the peer-to-peer environment, then the type of each peer would remain unknown; peers would select their providing peers randomly and contentions would also be resolved randomly. For this case, the success ratio among requested services per peer of each of the two types is depicted in Figure 1. (This figure as well as similar figures to follow express the evolution of the efficiency metrics defined under study with time.) Notice that the efficiency per egotistic peer is greater than that per altruistic one, while both are very low. This can be explained as follows: Egotistic peers constitute $90 \%$ of the population. Thus, there are very few altruistic peers, which are the ones providing most of successful services. However, an altruistic peer cannot serve herself, thus having a small disadvantage compared to an egotistic peer.

Next, we assume that there is a distributed reputation system employed that accurately calculates the reputation values of the peers. For ease of presentation, we first employ the success ratio among started services as an efficiency metric for our results. In the end of this subsection, we also study the success ratio among requested services and discuss on the suitability of both metrics. In the absence of reputation-based policies, each peer is assumed to follow the straightforward approach of using reputation (see Section 3 ). The curve denoted "max-random" in Figure 2 shows the success ratio among started services per altruistic peer following this straightforward approach. (Henceforth, in all figures "max" stands for the Highest Reputation policy. Also, we use interchangeably the expressions "random contention resolution policy" and "no policy".) Clearly, the success ratio among started services is greatly improved for altruistic peers when a reputationbased contention resolution policy (other than the random one) is also employed, as depicted by the other two curves of Figure 2. The opposite effect is observed for egotistic peers, as depicted in Figure 3. Combining Figures 2 and 3, it follows that the success ratio among started services of an altruistic peer is lower than that of an egotistic peer when no contention resolution policy is employed. Thus, the straightforward approach of using reputation is both inefficient and unfair.

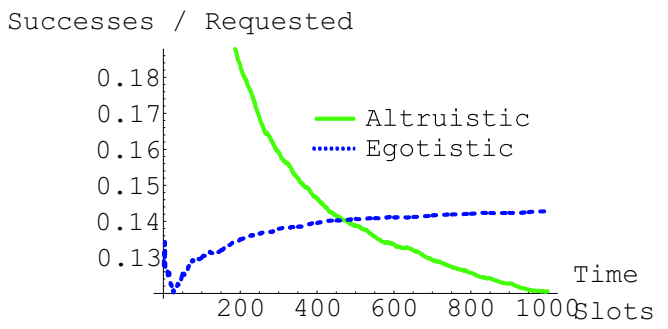

Figure 1. Success ratio among requested services per peer in the absence of a reputation system.

The success ratio among started services is improved (resp. deteriorated) for altruistic (resp. egotistic) peers in the case where the providing peers are selected according to the Compa- 


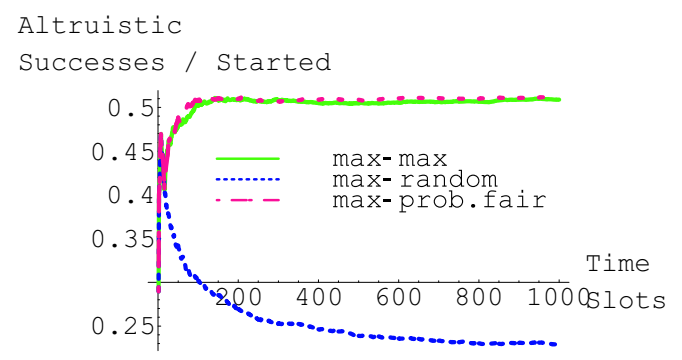

Figure 2. Success Ratio among started services per altruistic peer under the Highest Reputation provider selection policy combined with the three contention resolution policies.

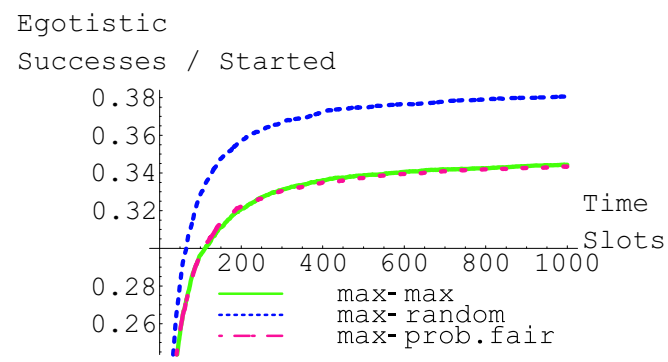

Figure 3. Success Ratio among started services per egotistic peer under the Highest Reputation provider selection policy combined with the three contention resolution policies.

rable Reputation policy for all contention resolution policies, as depicted in Figure 4 (resp. Figure 5). Also, notice that all presented contention resolution policies (including random selection) achieve similar success ratios for peers of the same type when this particular provider selection policy is employed. This is reasonably expected, since Comparable Reputation policy restricts contention only among peers having similar reputation values, thus rendering the selection of contention resolution policy immaterial. The resulting success ratio among started services per altruistic peer in the case where Black

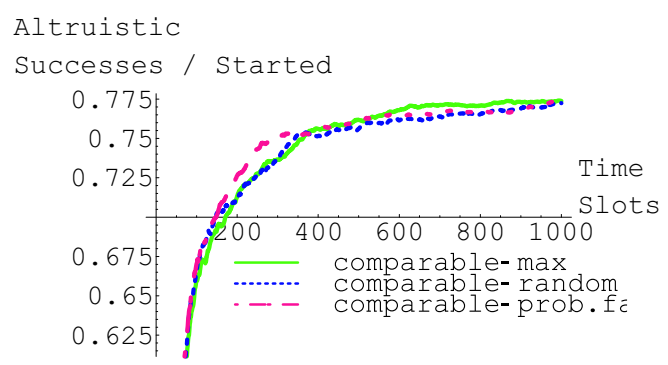

Figure 4. Success Ratio among started services per altruistic peer under the Comparable Reputation provider selection policy combined with the three contention resolution policies.

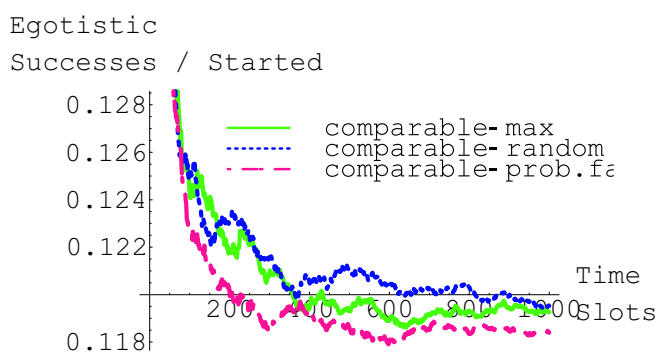

Figure 5. Success Ratio among started services per egotistic peer under the Comparable Reputation provider selection policy combined with the three contention resolution policies.

List selection policy is employed in the peer-topeer system is depicted in Figure 6 for the various contention resolution policies. Again, the achieved success ratios among started services of the Highest Reputation and the Probabilistically Fair contention resolution policies are close to each other. Thus, Highest Reputation and Probabilistically Fair contention resolution policies are almost equally efficient for each of the proposed provider selection policies that are combined with, while it is indeed beneficial and fair to employ one of them rather than the Random Selection policy. 


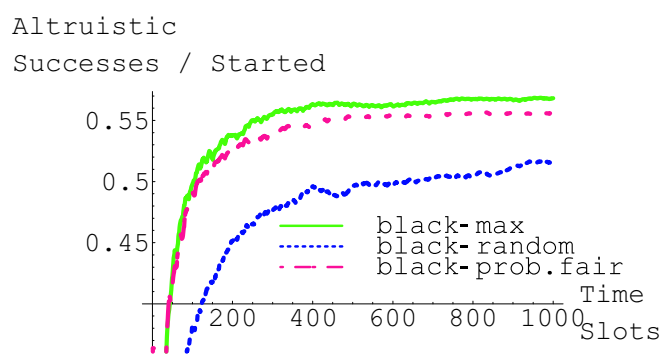

Figure 6. Success Ratio among started services per altruistic peer under the Black List provider selection policy combined with the three contention resolution policies.

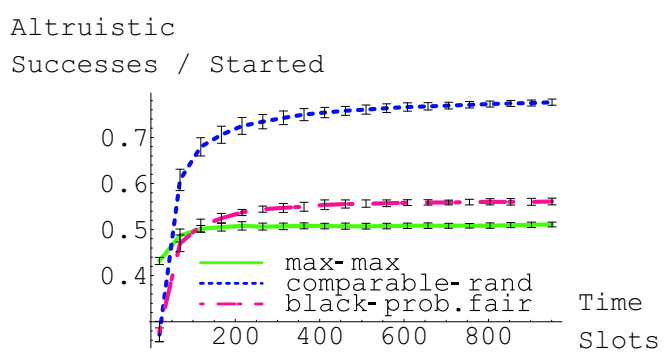

Figure 7. Success ratio among started services per altruistic peer under the most efficient pairs of reputation-based policies.

Next, we investigate the achieved efficiency of the various provider selection policies with each of them being employed jointly with the contention resolution policy that maximizes the achieved efficiency. Note that the choice of a contention resolution policy was immaterial for the Comparable Reputation provider selection policy, while for the Black List provider selection policy the Probabilistically Fair contention resolution policy was chosen to be combined with, rather than the Highest Reputation one, since both combinations lead to very similar efficiency for altruistic peers. The achieved success ratios among started services per altruistic peer for each pair of policies are depicted in Figure 7. Observe that Compa- rable Reputation outperforms the other two policies. This can be explained as follows: since reputation values are representative of peer types, under this policy a peer requests services only from peers of her type; thus, altruistic peers mainly interact with each other. Figure 7 (as well as Figure 8) depicts both mean values and confidence intervals over 10 simulation runs of the experiments. The fact that the confidence intervals are small and decreasing with time further corroborates the efficiency comparisons derived from the single-run results of previous figures. Note that

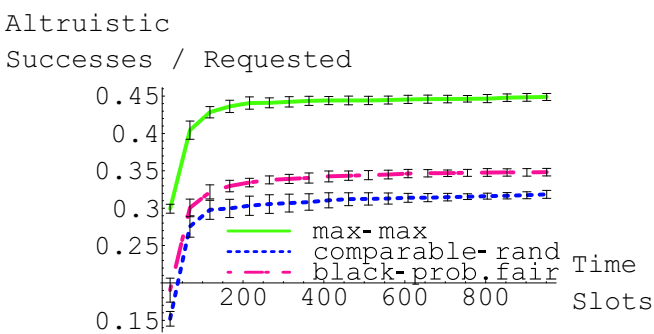

Figure 8. Success ratio over requested services per altruistic peer under the most efficient pairs of reputation-based policies.

the various provider selection policies perform differently regarding success ratio among requested services than success ratio among started services per altruistic peer, as depicted in Figure 8. In particular, the Highest Reputation provider selection policy outperforms the other two policies. This can be explained as follows: Under the Highest Reputation provider selection policy, the set of potential providers for a requesting peer is the entire population of peers. This does not apply for either the Black List or the Comparable Reputation provider selection policies. Thus, the number of the total started services per peer is not the same under all policies, as opposed to the number of total service requests. In our experiments, the set of altruistic peers is small (100 peers) and thus the resulting service availability for altruistic peers is limited under the Black List and the 
Comparable Reputation provider selection policies. Clearly, in a system where only a small number of peers belong to the high-performing type, finding the requested service at some providing peer, regardless of her type, is the most important issue. In this case, Highest Reputation provider selection policy is preferable to Comparable Reputation since only the former policy results in a high probability of finding the requested service. On the other hand, in a system with a large set of altruistic peers, the probability for the requested services to be found at a particular peer within this set is high, and thus success ratio among started services is the most important parameter of efficiency. The Comparable Reputation provider selection policy achieves the highest efficiency in this case. In certain cases of services, the most important efficiency parameter is the ratio of successfully provided services over the total number of started services; e.g., in case of a software sharing service where it is important to avoid malicious software. In other cases, the most important efficiency parameter for peers is the ratio of successfully provided services over the total service requests, e.g. sharing amusing content files among peers.

To summarize, the most efficient reputationbased policy pairs discriminate the value offered to peers of each type in a fair way. Moreover, if a peer can determine her type prior to entering in the peer-to-peer system, then these policy pairs provide the incentives for a peer to select being of the high-performing type.

\subsection{Short-lived Peers}

In this subsection, we analyze the efficiency of reputation-based policies for very short-lived peers. In particular, we assume that the population of peers is renewed with a rate $\lambda=30$ peers/time slot, i.e. three times as fast as in the previous experiments. Recall that, according to our simulation model, when a peer leaves the system a new peer of the same type enters with a new pseudonym and a clean record. In case of shortlived peers, the cycle of reputation information is short, and thus the effectiveness of each reputation policy is tested under "tighter" conditions. In Figure 9, depicted are the achieved success ra-

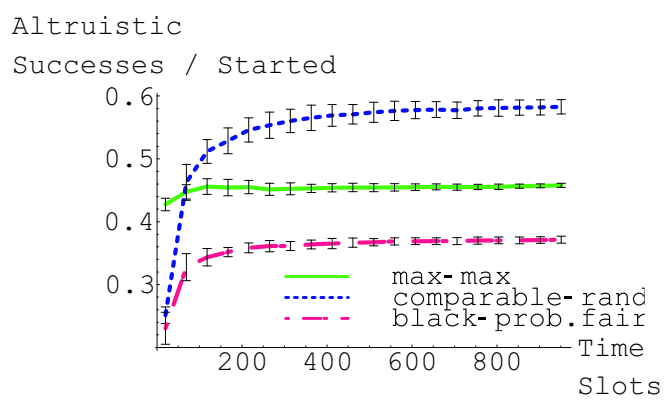

Figure 9. Success ratio for short-lived altruistic peers under the most efficient pairs of reputationbased policies.

tios among started services for altruistic peers for each of the most efficient pairs of provider selection policies. Again, depicted are mean values and confidence intervals over 10 simulation runs. The Comparable Reputation provider selection policy still achieves the highest success ratio. Furthermore, the success ratio of Black List provider selection policy is lower than that of the Highest Reputation one. Black List is not so effective for short-lived peers, which is reasonably expected, because peers with low performance may not be revealed and may falsely be selected as providing ones.

\section{4. "Milking" Strategies}

So far altruistic peers were supposed to follow the same fixed strategy in performing the peerto-peer protocol efficiently, even in the last time slots of their lives. This can be reasonably expected if this strategy can be materialized without excess cost of the effort undertaken by altruistic peers. Otherwise, during the last service provisions of their lives, such peers may have the incentive to rely on their high reputation values and exert no effort, i.e. to "milk" their reputation [10]. In this subsection, we experiment on the incentives provided by the proposed reputationbased policies to altruistic peers that intend to follow "milking" strategies. In particular, we run two coupled peer-to-peer systems (modeled as described in Subsection 5.1) that only differ in the 
following: a specific altruistic peer is taken to follow the regular altruistic strategy in the first system and a "milking" strategy in the second, whereby she exploits her reputation without exerting effort for providing services for a constant number of time slots before the end of her estimated lifetime. (Recall that each peer "lives" in the peer-to-peer system for a duration that is exponentially distributed with mean rate $N / \lambda$ ). The population of the system is renewed with rate $\lambda=10$ peers/time slot, while Beta aggregation of ratings is employed since the system is very dynamic. When the aforementioned specific peer is replaced, the new entrant follows the above strategy. We count the number of services provided successfully to this peer in each of the systems during the "milking" periods of a large number of her consecutive "lives". The percentage difference of these two numbers is depicted in Figure 10 for each of the most efficient pairs of reputationbased policies as well as for the straightforward policy. It should be noted that, as the number of milking time slots increases, the various curves clearly converge to the respective asymptotic values. Comparable Reputation is the providerselection policy that deals more effectively with the "milking" strategy, as it leads to the highest reduction of value during the milking periods for the peer adopting this strategy. ("Value" refers to the number of successfully received services.) Moreover, all three pairs of reputation-based policies reduce significantly the potential for milking of reputation, since they lead to high reductions of value (between $30 \%$ and $50 \%$ ), thus providing the right incentives to peers. On the contrary, the straightforward approach leads to almost the same value for a specific peer regardless if she "milks" her reputation or not, thus making "milking" attractive due to the associated elimination of the provision cost.

\subsection{Dynamic Strategies}

In this subsection, we investigate the effectiveness of reputation-based policies in providing incentives for high performance to peers following individually rational strategies. Each rational peer is assumed to seek only to maximize her own net benefit, i.e. her individual efficiency

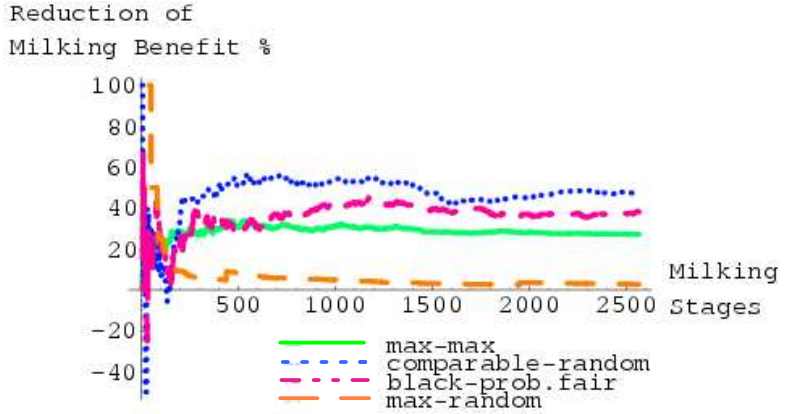

Figure 10. Percentage reduction of cumulative value (number of successfully received services) for an altruistic peer that "milks" her reputation under the most efficient pairs of reputation-based policies and under the straightforward approach.

while exerting the lowest possible effort for attaining this efficiency. For this purpose, we consider long-lived peers that enter the peer-to-peer system initially employing a high or a low success probability for providing services, and following the dynamic strategy described in Figure 11.

The dynamic strategy proposed in Figure 11 is incentive-compatible for rational peers to follow. According to this strategy, each peer periodically measures her received utility (i.e., the number of services successfully obtained) at the end of a certain service period in which she has a certain probability $s$ to provide successfully her services. The peer varies her probability $s$ depending on the impact of her previous actions on her own utility. Similarly to the simulation model of Subsection 5.1, initially $10 \%$ of peers succeed in service provision with probability 0.9 and the rest of them succeed with probability 0.1 respectively. The difference, however, with this model is that peers will not retain these probability values. In particular, according to the strategy of Figure 11, in the beginning a peer randomly chooses to increase or decrease $s$ and observe the effect of this action in her utility. If her previous action significantly improved her utility, then she repeats this action for the next service 
period. If her previous action significantly decreased her utility she takes the opposite action for the next service period. If her previous action did not have a significant impact in her utility, then she retains $s$ for the next service period, if her previous action was a decrease; otherwise she decreases $s$. Notice that with this strategy each peer tries to attain the highest possible utility with the minimum service probability among those that achieve that utility. The population of the peer-to-peer system is not renewed $(\lambda=0)$, as our objective is to observe the equilibrium strategy for peers when different reputationbased policies are employed. As the probability of successful service provision is not fixed, Beta aggregation is used for the computation of reputation values. The evolution of the service prob-

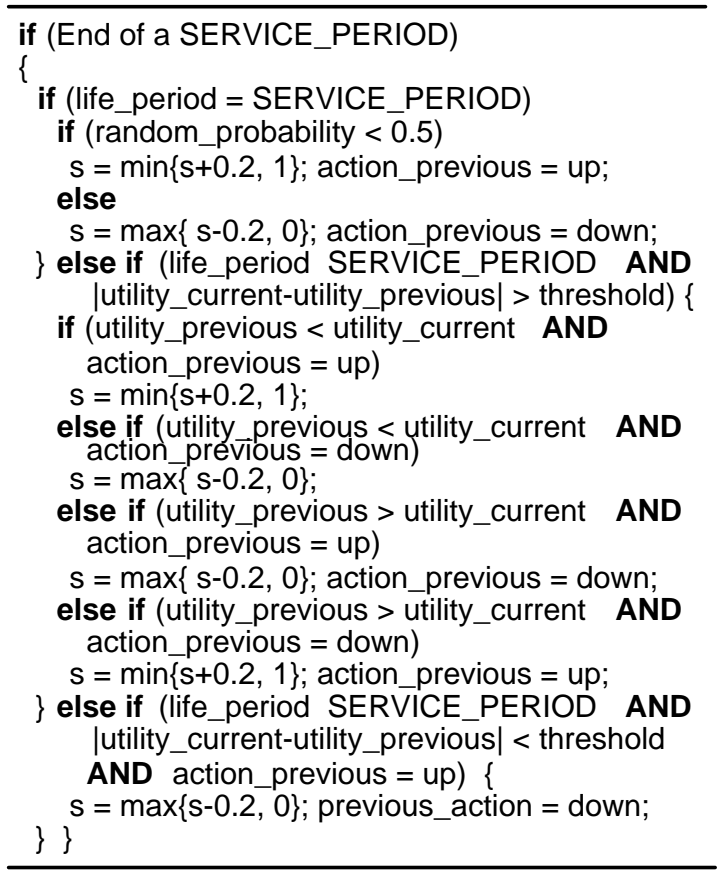

Figure 11. The individually rational strategy: If increasing $s$ was not beneficial, decrease $s$; otherwise, further increase $s$. If decreasing $s$ was harmful, increase $s$; otherwise further decrease $s$. ability of rational peers under the most efficient pairs of reputation-based policies is depicted in Figure 12. Clearly, if the straightforward reputation policy is employed, the probability of successful service provision of all peers converges to a low value (approx. 0.3). Also, the Black List provider selection policy fails to provide incentives for high performance, as in case of rational peers it is equivalent to selecting randomly the providing peer, because, almost all peers, being rational, avoid getting into the black list. Thus, in this case, incentives to peers for high service probability are only provided by the contention resolution policy. The fact that the "max-max" and the "comparable-random" reputation-based policies lead rational peers to evolve to higher service probabilities and close to 0.7 demonstrates that contention resolution policies are effective only when complemented with the right provider selection ones. Note that the service probability of rational peers does not evolve to 1 due to the step of increase/decrease (0.2) of the strategy of Figure 11 and the random phenomena involved in our model that make peers to oscillate their service probability around 0.7 . Also, there is high contention in the system that provides peers with no motivation to further improve (even closer to 1) their probability to provide services successfully.

\section{Implementation Issues for Reputation- based Policies}

Throughout this paper, we have assumed the existence of a mechanism, such as those in [3], [4] that enforce truthful reporting of feedback and non-manipulation of reputation values. Having resolved these issues, an important concern is whether reputation-based policies are employable in a real peer-to-peer system, where there may exist peers that can even "hack" their part of the peer-to-peer software (attempting to override the reputation-based policies), if they can gain in efficiency by doing so. Below, we discuss the applicability of reputation-based policies in practical cases. The incentives offered by the various policies are a key factor for this. First, note that contention resolution policies have no direct im- 

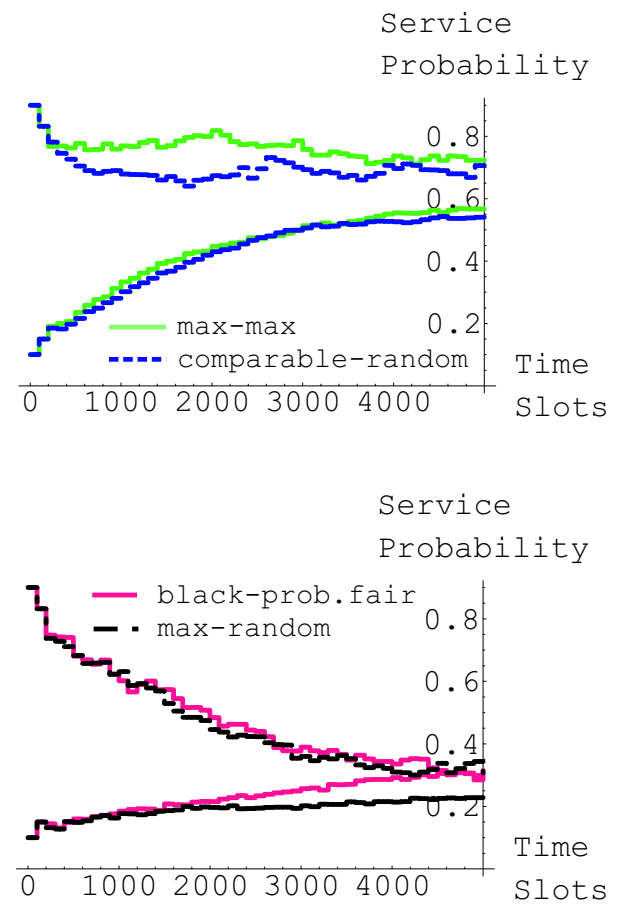

Figure 12. Evolution of successful service provision probability (denoted as service probability in the graphs) for initially high and initially low performing rational peers when a) "max-max" and "comparable-random" and b) "black-prob.fair" and the straightforward reputation-based policy pairs are employed.

pact to the efficiency of the providing peer himself. Thus, such a policy can be pre-configured in the peer's part of the peer-to-peer software without any further enforcement mechanism, as peers have no performance-related incentive to bypass or modify it. In fact, high-performing peers, who are the ones that generate the value in the peerto-peer system, have a long-term incentive to keep it (as in this way they receive higher value from the system). Even if some of the low-performing peers decide (for some strategic reason) to modify this part of their software, this will essentially have no impact to the efficiency enjoyed by the rest of the system, as only high-performing peers offer value in the system. Regarding provider selection policies, the incentives provided by Highest Reputation are compatible with the short-term objective of each individual peer to maximize the utility she obtains when she is provided with a service. Black list provider selection policy can also be easily applied, storing a warning flag in the reputation holder(s) of each peer. This flag is only announced, stating whether a peer belongs to the black list, in order for other peers to avoid transactions with her. These policies have to be combined with a contention resolution policy in order to be effective. As already discussed, this is practically feasible.

Comparable Reputation provider selection policy is effective regardless of whether or not it is combined with a contention resolution policy (see Subsection 5.2). However, this policy is trickier to apply, because each peer would have the incentive to try to illegitimately transact with highreputed peers. Below, we propose an approach to circumvent this: We safely assume that peers will always tend to select among the permissible providing peers the one that has the highest reputation, if they have the necessary information. Consider that the peer-to-peer system is divided into disjoint groups of peers, each constituting an independent subsystem, in the sense that peers in a group cannot transact with peers of another group. Each group comprises peers of similar reputation values and thus of similar expected performance levels. This feature should be enforced by the software. Each peer uses a unique pseudonym. New entrant peers in the peer-topeer system become members of the group that contains peers with the lowest reputation values. Peers are moved by the software to higher or lower groups according to the evolution of their respective reputation values. It is important to note that starvation is unlikely to happen for peers when Comparable provider selection policy is employed, because, in a real peer-to-peer system many new peers are expected to be in transit from the lower layers to the one that matches their type. Finally, peers are expected to be of various types and thus in large peer-to-peer systems many peers are expected to belong in each 
layer.

\section{Randomized Aggregation}

In this section, we deal with the efficient aggregation of ratings in terms of communication overhead in a peer-to-peer system in the absence of a central authority. Under the approaches of [4] and [6] some peers, determined by a set of hash functions, are responsible for holding and providing upon request the reputation value of one or more other peers. Reputation values are calculated by means of proper functions for the aggregation of votes, such as those discussed in Section 2 . The votes sent by a peer are associated to the peers she has transacted with and are included in the calculation of her reputation. Thus, the number of feedback messages that have to be sent to the reputation holders per unit time is proportional to the mean rate at which a peer is served. The set of messages required to be sent for the proper update of reputation information may induce a significant traffic overhead to the underlay network of a peer-to-peer system. This overhead can be reduced by aggregating the ratings for the same providing peer that arise within a time period prior to their submission to the corresponding reputation holder(s). However, the achieved reduction would in general not be significant, because transactions of a certain peer with the same provider peer within reasonably small time periods are rare.

The impact of incomplete feedback on a seller's maximum attainable payoff in an e-market was analyzed in [10]. In this paper we go further, by proposing that a peer submits to the corresponding reputation holders only a small randomly selected subset of her ratings that constitute a small portion $p$ of her entire set of ratings. We investigate the impact of reducing the feedback information to the efficiency of the peers, which, contrary to the model of [10], function as both servers and clients. Thus, the total number of feedback messages sent is reduced by a factor of $p$. This aggregation approach can be realized as follows: following a transaction, the vote is sent to the corresponding reputation holder with probability $p$. The following questions are in order: How much this reduction of feedback affects the accuracy of the reputation values of peers and the effectiveness of the reputation mechanism combined with reputation-based policies? What are the values of $p$ that induce only small losses in the efficiency of the peer-to-peer system while achieving considerable reduction of the communication overhead?

Next, we investigate the above questions by means of simulation experiments. Specifically, we consider the simulation model of Subsection 5.1, having each peer to collect with probability $p$ her feedback information per transaction and aggregate it locally for a short period of $T=15$ time slots, and then send it to the proper aggregators. In Figure 13(a), depicted are the average reputation values of altruistic and egotistic peers after 1000 time slots, as a function of the fraction $p$ of submitted ratings. Observe that the two types of peers are adequately differentiated with respect to their reputation values even for very small values of $p$ such as $10 \%$. The actual value of $p$ essentially does not depend on the lifetime per peer or on the request rate of other peers as long as a sufficient subset of feedback information can be aggregated for this peer early enough in her lifetime. Also, despite the reduced feedback information $(p=10 \%)$, reputation values converge fast with respect to time, as depicted in Figure 13(b). In Figure 14, depicted are the achieved success ratios among started services for altruistic and egotistic peers as the fraction $p$ of submitted ratings increases, under the combination of the Highest Reputation provider selection policy and the Probabilistically Fair contention resolution policy. Notice that even for small values of $p(\sim 10 \%)$ the achieved success ratios are very close to the values achieved when the complete ratings' information is used for the calculation of reputation values under the same pair of reputation-based policies. These values are depicted by horizontal dashed lines " $p=1$ " and are obtained from Figures 2 and 3.

Randomized aggregation of feedback information can be implemented along with a credibility mechanism, such as the ones described in [3], [4], for enforcing credible reporting. Aggregators of reputation values would poll peers for feedback with a certain probability per transaction. Even 

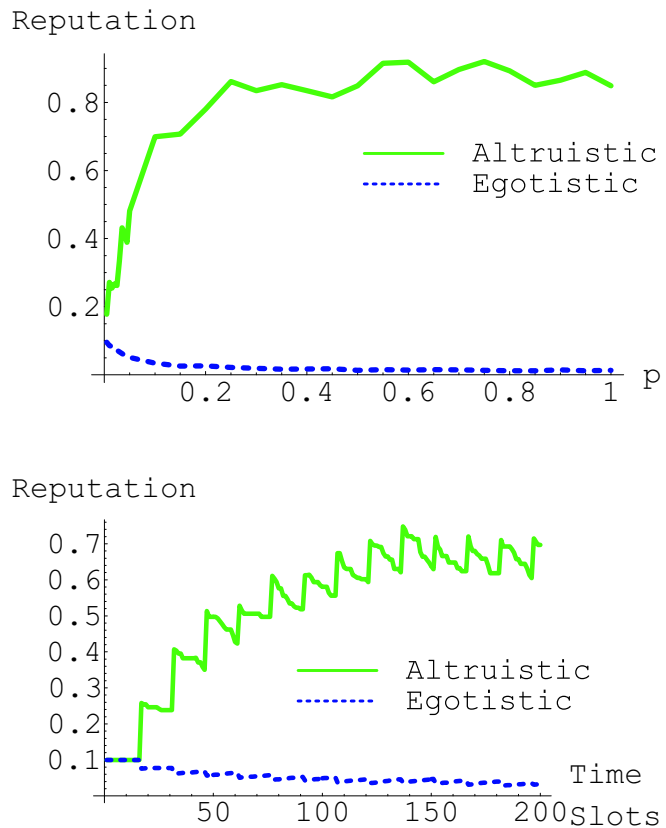

Figure 13. a) The limiting (with respect to time) values of reputation per altruistic and egotistic peer as a function of $p$. b) Reputation values converge fast with respect to time for $p=10 \%$.

if no such polling mechanism is employed, it is expected that peers are willing to send some part of feedback information. However, most of the nonreported feedback can be reasonably expected to be positive; this is one of the most pessimistic circumstances to evaluate our approach. Assuming for simplicity that all negative feedback is indeed reported, we have shown experimentally that a small percentage $p^{+}$of positive feedback information is enough for the accurate computation of the reputation values of peers. In particular, for $p^{+} \simeq 25 \%$ or greater, the limiting (with respect to time) reputation values of altruistic and egotistic peers are above 0.8 and below 0.05 respectively. This implies that the feedback peers are willing to send on their own is sufficient for reputation accuracy and efficient in terms of communication overhead. Sending a small randomly selected sub-

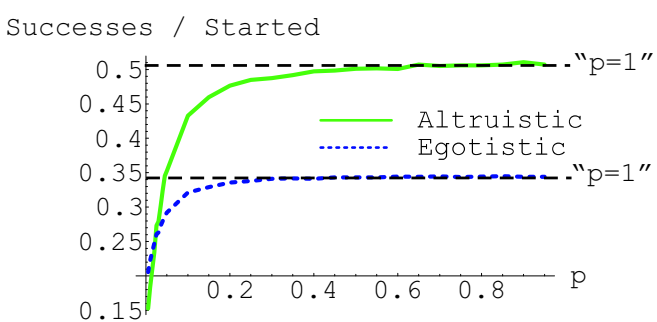

Figure 14. Success ratio among started services per peer for the various values of $p$.

set of the feedback information also serves the objective of privacy. To see this, consider that by attaching the reputation values to pseudonyms the desirable inherent characteristics of peer-topeer systems, such as anonymity, are preserved. However, ratings provide feedback to reputation holders for the complete history of transactions of a peer. Thus, privacy concerns are still applicable. Our proposed randomized aggregation approach limits considerably these concerns, as feedback about only a small random part of the entire history of the transactions of each peer is revealed. Also, note that this approach is still applicable in the case that peers employ dynamic strategies, as revealed by simulation experiments that are not presented in this paper. In such a case, the effect of reputation-based policies arises more slowly.

\section{Comparison with Related Work}

In this paper, we showed that the straightforward policy (i.e. to employ reputation only to select the highest-reputed providing peer) is not fair for high-performing peers and it does not provide the right incentives to peers for service provision. In [7], it is argued that, when a reputation metric is in place, high-reputed peers may become the bottleneck for service provision and thus the resulting efficiency for other peers may be reduced, which seems a similar argument to 
ours. However, in this paper, we have gone considerably further by showing that the selection of the highest-reputed peers for service provision is an effective policy if the contention for the resources of high-performing peers is resolved according to the reputation of the requesting peers. Also, it is important to note that in reality the highest-reputed peers are not expected to concentrate the whole amount of service requests due to the diversity in service availability and in service requests of peers. In [11], a bidding mechanism is proposed for the proportionally fair allocation of the resources of a providing peer to the contribution values of the requesting peers in the peer-topeer system. This policy is analogous in its effect to our "Probabilistically Fair" contention resolution policy. However, as shown in Subsection 5.5, the effectiveness of this policy is increased, if it is appropriately combined with a proper provider selection policy, which is not considered in [11].

Also, [8] investigates the evolution of different species of peers that follow different strategies regarding offering content while reputation metrics for usage and service are employed. The Highest Reputation contention resolution policy is employed for the allocation of the bandwidth of a providing peer in the limit of link capacity. On the other hand, providing peers are selected randomly. Even though the evolution of the various species is done in favor of the high-performing peers, the effectiveness of this policy would be increased, if it were appropriately combined with a provider selection policy, as shown in Subsection 5.5. Also, certain provider selection reputationbased policies were used for experiments in [6], [7], as we mentioned in Section 4.

In [12], a different dynamic strategy for service provision is described that offers high probability of successful service provision to clients that contribute higher value to the peer-to-peer system than they receive from it. It is not incentive compatible for providing peers to follow this strategy and thus it was not used in our experiments with dynamic strategies.

Finally, in [13], another dynamic strategy for service provision is proposed. This strategy has similar objectives to the strategy presented in Subsection 5.5, i.e. each peer aims to exert the minimum effort (probability of successful service provision) that results in the maximum success rate. However, this strategy is not incentive compatible as it suggests that peers increase their service probability when they receive less efficiency from the system. Also, in [13], a sampling of reputation values of peers is proposed in order to estimate certain metrics related to the systemwide distributions of provision and consumption of peers. This method is specific to the aforementioned strategy and it is not comparable to our randomized aggregation approach.

\section{Concluding Remarks}

In this paper, first, we have shown that the straightforward approach for peers to exploit the reputation metrics in peer-to-peer systems may be unfair and may not provide the right incentives to high-performing peers regarding successful service provision. We have introduced two dimensions of reputation-based policies, namely provider selection and contention resolution. We have experimentally proved that both have a significant impact on the efficiency as well as on the provision of the right incentives to peers regarding successful service provision. This conclusion holds true both when peers follow static strategies and when peers employ individually rational strategies regarding their performance in service provision. An important question is which policy is recommended in practice: Comparable Reputation provider selection policy achieves for highperforming peers the highest success ratio among started services (regardless of the contention resolution policy combined with). Highest Reputation policy employed simultaneously for provider selection and for contention resolution achieves for high performing peers the highest success ratio among requested services. The selection of the most appropriate policy pair between them depends on the specific service being offered in the peer-to-peer environment, as explained in Subsection 5.2. Both policy pairs are equally effective: i) in providing incentives to rational peers for high probability of providing services successfully, and ii) in limiting the potential for undesired exploitation of reputation, i.e. "milking". Also, we 
have explained how the reputation-based policies can be employed in a real peer-to-peer system. From another perspective, we have experimentally proved that a small randomly selected subset of the ratings' feedback is sufficient information for the fast and accurate calculation of the reputation values. This result holds even if the population of peers is renewed with a high rate. We have argued that the submission of such feedback by peers is incentive compatible, i.e. there is no need for any special mechanism to enforce it. The communication (and processing) overhead would thus be significantly reduced. In future work, we intend to investigate applicability and effectiveness of our reputation-based policies in an e-commerce environment, where peer transactions also involve payments. We believe that this constitutes an interesting and challenging direction for research.

\section{Acknowledgements}

We thank Costas Courcoubetis, Huw Oliver, Ben Strulo and the other members of the consortium of IST project MMAPPS for useful discussions on the subject of this paper. We also thank the anonymous referees for their valuable comments.

\section{REFERENCES}

1. C. Dellarocas, The Digitization of Word-ofMouth: Promise and Challenges of Online Feedback Mechanisms, Management Science, October 2003.

2. A. Jøsang, S. Hird, E. Faccer, Simulating the Effect of Reputation Systems on e-Markets, in; Proc. of the 1st International Conference on Trust Management, Crete, Greece, May 2003.

3. T. G. Papaioannou, G. D. Stamoulis, An Incentives' Mechanism Promoting Truthful Feedback in Peer-to-Peer Systems, in; Proc. of IEEE/ACM CCGRID (Workshop on Global P2P Computing), May 2005.

4. K. Aberer, Z. Despotovic, Managing Trust in a Peer-to-Peer Information System, in; Proc. of the 10th International Conference on Information and Knowledge Management (CIKM01), New York, November 2001.

5. T. G. Papaioannou, G. D. Stamoulis, Effective Use of Reputation in Peer-to-Peer Environments, in; Proc. of IEEE/ACM CCGRID (Workshop on Global P2P Computing), April 2004.

6. S. D. Kamvar, M. T. Schlosser, H. GarciaMolina, EigenRep: Reputation Management in Peer-to-Peer Networks, in; Proc. of the WWW Conference, Budapest, Hungary, May 2003.

7. K. Ranganathan, M. Ripeanu, A. Sarin, I. Foster, 'To Share or not to Share' An Analysis of Incentives to Contribute in Collaborative File Sharing Environments, in; Proc. of the Workshop on Economics of Peer-to-Peer Systems 2003, Berkeley, CA, USA, June 2003.

8. J. Hwang, C. H. Lee, Agent-based Modeling for Differentiated Admission in P2P Systems Using Evolutionary Game Theory Focused on Ownership Reputation, in; Proc. of the Workshop on Economics of Peer-to-Peer Systems, Harvard, MA, June 2004.

9. E. Adar, B. Huberman, Free riding on Gnutella, FirstMonday, vol. 5, no. 10, October 2000 .

10. C. Dellarocas, Sanctioning Reputation Mechanisms in Online Trading Environments with Moral Hazard, MIT Sloan School of Management, Working Paper No. 4297-03, July 2004.

11. T. B. Ma, S. C. M. Lee, J. C. S. Lui, D. K. Y. Yau, A Game Theoretic Approach to Provide Incentive and Service Differentiation in P2P Networks, in; Proc. of ACM SIGMETRICS/PERFORMANCE, June 2004.

12. K. Lai, M. Feldman, I. Stoica, J. Chuang, Incentives for Cooperation in Peer-to-Peer Networks, in; Proc. of the Workshop on Economics of Peer-to-Peer Systems 2003, Berkeley, CA, USA, June 2003.

13. H. T. Kung, C.-H. Wu, Differentiated Admission for Peer-to-Peer Systems: Incentivizing Peers to Contribute their Resources, in; Proc. of the Workshop on Economics of Peer-toPeer Systems, Berkeley, CA, USA, June 2003. 